\title{
FAMILIA Y ESTADO: UNA RELACION A EXAMEN
}

Para comprender las actuales relaciones entre el Estado y la familia y la forma en que aquél incide sobre ésta debemos analizar el proceso histórico que dio origen a los diferentes modelos familiares que hoy existen. Una serie de fenómenos sociales que se produjeron a partir del siglo xv y culminaron con el proceso de industrialización son el marco y el punto de referencia para comprender las transformaciones que sufrió la familia medieval hasta dar origen a la familia moderna. De ellos, cinco parecen cruciales.

En primer lugar, la aparición del individuo como sujeto y protagonista de la actividad económica, ya que puede poseer una propiedad de la que disponer libremente o puede vender su fuetza de trabajo en el mercado. Así, el individuo aparece dotado de capacidad legal para entablar diálogo con los poderes estatales. Este individuo político y económico se convierte al mismo tiempo en un ser subjetivo, con necesidades emotivas y racionales que debe buscar satisfacer.

En segundo lugar, se produce la tecalificación de un espacio social único en dos nuevos espacios: el lugar de lo privado y el lugar de lo público. Si bien ambos espacios ya existían, la vida de la comunidad los enlazaba. Así, la familia cumplía funciones sociales y políticas importantes, aunque poseía una dimensión privada. $\mathrm{La}$ industrialización va a diferenciar las actividades públicas de las privadas, convirtiéndolas en dos ámbitos separados. 
En tercer lugar, la recalificación del espacio económico - que a su vez supone la recalificación de la familia - con la emergencia de dos nuevos espacios: el de la producción de bienes y servicios y el de la reproducción de la vida y de las energías humanas. En la familia se concentratán las actividades de reproducción y la prestación de ciertos servicios. El ámbito económico público será la esfera de la producción de bienes y servicios.

En cuarto lugar, la ordenación del tiempo con una división entre trabajo y ocio, para la que ya no es útil el calendario tradicional. El ocio, antes considerado como la posibilidad de crear cultura, esparcimiento y vida personal, se convierte en algo residual y condenable. Se eleva el valor del trabajo, a partir del cual se desarrolla una ética nueva. Así, las actividades productivas pasan a recibir más prestigio social y surge la contraposición entre ocio y trabajo en la que se deja poco lugar al ocio, sancionando a quienes no están dispuestos a acatar la moral laboral.

Finalmente, la consolidación del Estado moderno como ordenador de los espacios emergentes. El Estado, al comienzo sólo detenta algunas funciones reguladoras de la vida social. Pero, en la medida en que el proceso de industrialización se consolida estas funciones se van ampliando, de modo que el Estado interviene de una forma cada vez más activa en casi todos los ámbitos de la vida social.

Este proceso de transformaciones sociales conduce a una serie de modificaciones de la familia. Para analizar los cambios producidos es importante destacar cuatro etapas, cada una de las cuales genera nuevas demandas y nuevas formas de relación dentro de la familia y entre ésta y la sociedad.

a) Siglos xv al xvir: Marcan el paso del feudalismo al metcantilismo. La familia amplía el alcance de su actividad productiva en la medida en que además de bienes de uso genera mercancías. Existe una división sexual del trabajo, pero tanto mujeres como varones participan en la producción.

b) Siglo xvirr: Aparece la manufactura. Mujeres, niños y varones salen de sus casas para integrarse en la producción de mercancías en un lugar nuevo. La vida de las personas queda reducida al tiempo de trabajo y la familia es apenas el lugar de la reproducción biológica.

c) Siglo xIx: La fábrica. Supone un incremento de la productividad del trabajo de tal alcance que hace inútil la participación de las mujeres y los niños: ellas vuelven a casa y los niños van a la escuela. Éste es justamente el momento en que podemos hablar de un lugar de lo privado $\mathrm{v}$ otro de lo público. El entorno de las mujeres y los niños, la afectividad, la reproducción de la fuerza de trabajo conciernen a lo privado; el trabajo asalariado, las luchas políticas y sindicales, conciernen a lo público.

d) Siglo $\mathrm{xx}$ : Las mujeres hacen suyo el ideario de los derechos indi- 
viduales y luchan para que se les hagan extensivos. La primera guerra mundial actuará como acelerador porque la incorporación de los varones al frente permite a las mujeres acceder al trabajo asalariado y a los servicios colectivos. En el período entre las dos guerras se produce un reflujo en su participación laboral. No obstante, la lucha de las mujeres reforzada por su incorporación a la vida pública en los momentos de guerra produce la modificación del marco legal que ahora reconoce que la mujer también es sujeto de derechos. En la actualidad esta lucha toma el sentido de que estos derechos legales se conviertan en una realidad práctica.

En los tres primeros momentos la fuerza motriz hegemónica es de carácter estrictamente económico. En el cuarto, correspondiente al siglo $\mathrm{xx}$, el vector de cambio es la conciencia personal de las mujeres. Éstas, impregnadas del ideario de la revolución burguesa, luchan por conseguir el estatuto de individuo. Es esto lo que produce las transformaciones de la familia que podemos observar hoy en la mayoría de las sociedades occidentales.

Hasta aquí se han descrito los elementos sociales y políticos que configuran el marco en el cual se han desarrollado los modelos familiares que surgen en el período histórico que va desde el mercantilismo hasta la época contemporánea. Con las necesarias simplificaciones se puede hablar de tres modelos de familia: la feudal, la burguesa y la moderna.

La familia feudal se caracterizaba por ser amplia (incluía a muchos parientes) y porque en ella coincidian las funciones de producción, reproducción y consumo. La división del trabajo se basaba en la edad y el sexo, aunque todos los miembros de la familia participaban en las tareas productivas. El concepto de niñez no existía tal como se entiende hoy, pues no se diferenciaba al niño del adolescente, salvo para considerarlo un adulto en potencia, y la jerarquía estaba claramente encarnada en los hombres más viejos. Las familias cumplían al mismo tiempo funciones sociales de importancia: en el caso de la nobleza, las alianzas de poder eran familiares.

La familia burguesa aparece en la medida en que las relaciones económicas y políticas del feudalismo dan paso al capitalismo. Al atenuarse los lazos feudales y emerger el individuo, la familia reduce los lazos de parentesco a los cónyuges y sus hijos. Se convierte, al mismo tiempo, en el sitio donde surge la privacidad definida en dos áreas: la propiedad y el trabajo individual privado por un lado, y las relaciones personales privadas entre sus miembros, por otro. En términos ideológicos esto implicó la valoración de los atributos de disciplina, orden y ahorro necesarios para la acumulación del capital. En términos políticos implicó la demanda de democracia, entendida como un derecho de los jefes de familia. Al mismo tiempo que se 
produce este cambio surge el Estado que reemplaza al poder basado en el parentesco y las alianzas familiares.

Al comienzo, durante la época mercantil, la familia burguesa mantuvo la unidad entre producción y reproducción. Los miembros de la familia, más sirvientes y aprendices, participaban en el proceso productivo bajo la autoridad paterna. La familia comienza a diferenciarse del resto de la sociedad cada vez más, al tiempo que se consolida el poder del jefe de familia. Sin embargo, mantiene sus funciones económicas. Pero con la aparición de las industrias y el consecuente proceso de industrialización, esta situación se modifica fundamentalmente.

La producción de bienes se traslada a las fábricas mientras que la familia se convierte en el lugar donde se realizan las tareas de reproducción, las de afectividad y las relaciones personales. Esta separación de funciones se convierte en una división del trabajo por sexo: los hombres salen a trabajar a las fábricas y las mujeres permanecen recluidas en el hogar, dedicadas al trabajo doméstico y al cuidado de los hijos. Esta división sexual del trabajo aparece primero en la clase burguesa. Los campesinos, convertidos en proletarios, viven un proceso de desintegración aguda de la familia. Hombres, mujeres y niños participan del trabajo productivo en las fábricas, con horarios extensos que no dejan tiempo libre para la vida personal, familiar o el ocio. Pero, en la medida en que el capitalismo se afirma y las organizaciones sindicales logran que aumenten los salarios y disminuyan las horas de trabajo, las mujeres de la clase obrera retornan al hogar y el modelo de familia burguesa se extiende a toda la sociedad.

Con la consolidación de la industrialización surge la familia moderna, que se caracteriza por la división entre las tareas de producción y reproducción. La familia se convierte en el lugar donde se realizan los servicios relacionados con la reproducción y en el centro del consumo. Se consolida, así, la división entre el mundo público y el privado. La economía y la política se convierten en actividades públicas, mientras que la reproducción, los sentimientos y las relaciones personales son actividades privadas que se desarrollan en el marco de la familia.

Durante el siglo xIx el Estado interviene por primera vez en la familia. Se trata de recomponer la familia feudal desarticulada y sustituirla por la familia burguesa, especialmente en lo que se refiere a la consolidación de la autoridad paterna y sus rasgos patriarcales. El Código Civil napoleónico, que sirve de modelo al de muchos otros países, establece los papeles y funciones que los miembros de la familia deben desempeñar, al mismo tiempo que señala claramente la posición subordinada de las mujeres en ella.

Nos encontramos, entonces, que en los países industrializados o en vías de industrialización, se impone finalmente lo que los sociólogos han 
denominado «familia moderna». Pero tras esta etiqueta se pueden distinguir diferentes tipos y funciones familiares que responden a las formas de industrialización y al tipo de Estado que estos países han desarrollado.

Un primer tipo de familia moderna se caracteriza por una división rígi$\mathrm{da}$ de los roles sexuales y por su estructura decididamente patriarcal y autoritaria. Los hombres tienen acceso preferencial al trabajo asalariado y poseen derechos políticos y sociales. El Estado les concede la autoridad sobre los demás miembros de la familia a través de la legislación y la práctica. Es el modelo autoritario, heredero directo de la familia burguesa.

Un segundo tipo de familia moderna se caracteriza por la misma división sexual del trabajo pero, en este caso, las mujeres han adquirido los mismos derechos que el hombre. Comparten la autoridad sobre los hijos, que se hace menos rígida, y los derechos sobre los bienes que la familia posee. Al mismo tiempo, se liberaliza la concepción de las relaciones sexuales entendidas, ya no sólo en su función reproductiva, sino como parte de la relación afectiva entre los cónyuges. El vínculo familiar ya no es indisoluble, pues en la medida en que se pone énfasis en la afectividad, cuando ésta desaparece se puede optar por el divorcio. A este segundo tipo de familia moderna la llamaremos familia democrática.

Ambos tipos de familia tienen en común la separación radical entre las actividades masculinas y femeninas, dando origen a dos universos claramente diferenciados, cada uno con su propia lógica de funcionamiento. Sin embargo, a diferencia de lo que los sociólogos han tendido a afirmar, no es verdad que esta separación implique que la familia ha perdido funciones económicas. En realidad, en la familia se siguen produciendo los servicios que permiten la reproducción de la fuerza de trabajo, tanto la biológica (procreación y cuidado de hijos) como la cotidiana. Esta última presupone que en la familia se realizan las actividades domésticas que permiten reponer cada día las energías y el acondicionamiento del trabajador.

Lo importante es señalar que esta función económica ya no es desempeñada por todos los miembros de la familia: se convierte en la actividad de las mujeres. Son ellas, también, las depositarias de los sentimientos y la afectividad. La implantación del capitalismo hizo surgir al individuo la necesidad de relaciones personales y de la subjetividad. Pero los valores de la sociedad capitalista y su énfasis en la competencia, la burocracia y la impersonalidad, hacen que esta subjetividad no pueda desarrollarse más que en la familia. La alienación exterior es compensada, así, por las relaciones familiares.

Esta separación de los roles sexuales, masculinos los de las actividades públicas y femeninos los de las privadas, produce dos tipos de personalidades diferenciadas. $\mathrm{El}$ hombre desarrolla sus actividades en diferentes 
esferas que exigen formas diferenciadas de comportamiento: es trabajador y ciudadano (público) y padre de familia. Las mujeres, en cambio, son esposas, amas de casa y madres, tareas todas desempeñadas en el hogar. Los valores y normas que orientan estas tareas son diferentes: los roles desempeñados en el mundo público se caracterizan por la eficiencia técnica y la impersonalidad; los que se realizan en el seno de la familia por la afectividad y la falta de control técnico, ya que las mujeres pueden desempeñar las tareas domésticas en la forma y con el ritmo que más les convenga. Los hombres desarrollan primero una personalidad moderna, es decir, la capacidad de adecuarse a distintas circunstancias sociales. Las mujeres permanecen en una situación doble, premoderna, pero vinculadas indirectamente al mundo moderno. Se generan así los dos universos señalados, con lo que el ámbito social de los hombres y el de las mujeres (y sus personalidades) aparecen como polos opuestos y complementarios.

Hay, finalmente, un tercer tipo de familia moderna, la familia igualitaria. En este caso, la mujer se ha incorporado al trabajo productivo asalariado, pero manteniendo las funciones de madre, esposa y ama de casa. Su implantación es más reciente y se ha producido en las sociedades en que era necesaria la aportación de las mujeres a la fuerza de trabajo. Se le abren, por tanto, las puertas del mercado de trabajo, aunque sigue siendo la responsable de las funciones familiares que ya poseía. Se trata, entonces, de que la mujer ejecute una doble jornada de trabajo. El calificativo de «igualitaria» hace referencia al contexto ideológico en que surge este tipo de familia y no a las consecuciones en términos de igualdad entre los dos sexos, pues sigue siendo la mujer la que desempeña los servicios que se realizan en la familia. Lo que aparece como «igual» es la incorporación de la mujer a las actividades económicas asalariadas. Sin embargo, estudios recientes muestran que esta incorporación se hace en términos absolutamente desiguales: salarios inferiores para la misma tarea, acceso a las ocupaciones que tienen menor status social y económico, marginación de la política y de las instituciones de poder e incapacidad de competir debido a la existencia de la segunda jornada laboral. Por lo tanto, la denominación de familia igualitaria hace referencia a los supuestos del modelo y no a su realización. Analizaremos a continuación a qué se debe esto.

Dos hechos del siglo xx van a poner en cuestión el modelo autoritario de familia que el Estado había sancionado en todos los países capitalistas en el siglo xIx: las dos guerras mundiales y la existencia del movimiento sufragista, primera versión del feminismo. El sufragismo, al exigir la extensión de los derechos ciudadanos a las mujeres, cuestiona la reclusión de las mujeres en el hogar. Algunos sectores del sufragismo convirtieron la demanda por la abolición de la familia autoritaria en parte de sus reivindi- 
caciones. Estos movimientos tuvieron especial importancia en los países en que el capitalismo había ido de la mano del liberalismo ideológico y político. Así aparecía como inconsecuente con los presupuestos del liberalismo la exclusión de las mujeres de los derechos ciudadanos. Las sufragistas mostraban que el Estado no podía tener dos normativas distintas, una para los hombres y otra para las mujeres.

Las dos guerras mundiales forzaron, en la práctica, a romper el modelo de separación absoluta de los roles sexuales. La incorporación masiva de los hombres a la guerra hace necesario que las mujeres se incorporen a las fábricas, desempeñando unas tareas que hasta el momento se consideraban «masculinas». Finalizadas las contiendas, las mujeres son devueltas al hogar. Pero ya no se les puede negar los derechos individuales. Es así como entre la primera y la segunda guerra mundial se concede el voto a las mujeres en muchos países, al mismo tiempo que se les permite acceder a la educación superior y a los trabajos remunerados. Estos derechos se extienden, al final de la segunda guerra mundial, a todos los países democráticos.

Tenemos, así, la segunda intervención estatal que termina con el modelo autoritario de familia y lo suplanta por el democrático. Esto, sin embargo, sólo sucede en los países en que el Estado es laico, liberal y democrático. No sucede lo mismo en los países en que la Iglesia no se ha separado del Estado. Para todas las religiones, pero especialmente para la Iglesia Católica, el modelo de familia autoritaria es consustancial con su ideología sobre la mujer y su función social.

Esta segunda intervención estatal se produce en los países democráticos a partir del fin de la primera guerra mundial y se consolida después de la segunda. En los países de regímenes autoritarios o de influencia de la Iglesia Católica (España e Italia, por ejemplo) el proceso es mucho más tardío. Junto con la legislación que concede derechos a las mujeres, comienza un proceso paralelo de incorporación a la vida pública que trae como consecuencia la aparición de la familia igualitaria. Se produce entonces una nueva intervención por parte del Estado para garantizar la participación de las mujeres en la actividad económica; que se hace más importante a partir de los años sesenta y, en especial, a partir del Año Internacional de la Mujer y los acuerdos firmados por los gobiernos en la Convención de las Naciones Unidas (1975) para eliminar toda forma de discriminación sexual.

La intervención del Estado para posibilitar la incorporación de las mujeres a las actividades públicas responde, por un lado, a las necesidades económicas y sociales de mano de obra femenina en el mercado laboral y, por otro, a la ideología de los grupos gobernantes. En el segundo caso se trata de gobiernos que sostienen en sus programas que deben eliminarse las diferencias por razones de sexo, dando acceso a las mujeres a las acti- 
vidades consideradas como «masculinas». Ya sea por una razón o por otra, el Estado debe proveer a la sociedad de los servicios que eran desempeñados por las mujeres en la familia, para liberarla de ellos. Esta intervención tiende a generalizar y consolidar el modelo igualitario de familia. Aunque se nota una tendencia en este sentido en todos los países industrializados, es en los países socialistas del Este y aquellos con años de gobiernos socialdemócratas, donde el proceso se ha desarrollado con más profundidad.

En los países socialistas del Este, la incorporación de las mujeres a la fuerza laboral era central para el desarrollo económico. Su ideología sobre la igualdad entre hombres y mujeres y el énfasis en la destrucción de la familia burguesa hace que el Estado asuma, o intente asumir, las labores realizadas por las mujeres en el hogar, de modo que éstas puedan incorporarse a las fábricas. Se crean, así, servicios colectivos que sustituyen, aunque parcialmente, el trabajo doméstico.

En los países socialdemócratas el desarrollo del Estado benefactor produce la asunción por parte del Estado de una serie de servicios antes producidos en el hogar. Para desempeñar estos servicios se contrata a mujeres. Este proceso adquiere importancia en los períodos de expansión económica en los que el mercado requiere mano de obra femenina. En los períodos de crisis económica, en cambio, se reducen los servicios y se despide a las mujeres. El Estado, en todo caso, ha desarrollado legislaciones y programas que permitan su plena incorporación al trabajo asalariado.

A pesar de los logros obtenidos en la igualación de derechos entre hombres y mujeres, la experiencia de estos países muestra que la introducción de este tipo de familia ha generado una serie de problemas.

En primer lugar, en todas las sociedades industriales el énfasis de la economía está en la producción de bienes y no en la de servicios. Así, cuando el Estado asume los servicios lo hace en forma impersonal, tecnocrática, burocratizada y de mala calidad. Esto tiende a deteriorar la calidad de la vida.

En segundo lugar, aunque el Estado puede asumir los servicios que antes prestaban las mujeres en la familia, no puede asumir los aspectos emocionales y afectivos de ésta. Las relaciones en el mundo público, caracterizadas por la impersonalidad y la racionalidad, son compensadas en la familia por relaciones personales. Frente a la competitividad y alienación del mundo exterior, la familia aparece como un refugio. Los mismos servicios prestados en la familia llevan este sello de personalidad que se pierde cuando el Estado lo asume.

Ahora bien, la incorporación de las mujeres al mundo público no presupone la revalorización de los servicios que ella prestaba en la familia. La valoración económica y social sigue dando prioridad a la producción de 
bienes. Las mujeres deben entonces soportar, por un lado, la presión del trabajo asalariado $y$, por otro, seguir siendo afectivas en el hogar. Se las carga con una doble jornada de trabajo, pero, además, con un doble código de conducta: competitiva en el trabajo externo y afectiva en la familia. Aunque en algunos casos el Estado ha intentado posibilitar que los hombres participen de las actividades domésticas, sobre todo en lo que concierne al cuidado de los niños, ello no se ha traducido en la práctica en la ruptura de las funciones sexuales.

Esto produce frustración en los miembros de la familia. En una sociedad patriarcal en la que los hombres son educados para asumir un modelo de conducta viril que, en última instancia, es un modelo de poder, las frustraciones que éstos sufren en el trabajo, donde son pocos los que pueden mandar, son compensadas con las cuotas de autoridad que detentan en la familia. Pero la familia igualitaria produce la rebelión de la esposa y los hijos ante esta autoridad, con lo que se acaba el soporte del hombre trabajador. Al mismo tiempo, la participación de la mujer en el trabajo en condiciones de inferioridad produce insatisfacción en las mujeres, lo que, sumado a la doble carga de trabajo, se traduce en frustración y alienación y produce un incremento de la violencia y los conflictos en el hogar.

Éstos son los problemas que se analizan en los artículos que se incluyen en este número de «Papers». M. Jesús Izquierdo y Rita Liljestrom analizan los problemas de la familia igualitaria en los países del Este y en Suecia. Laura Balbo estudia las situaciones que se presentan cuando el Estado asume los servicios y se produce una crisis económica. Inés Alberdi analiza las transformaciones legales que ha sufrido la familia española y la transición de la familia autoritaria a la familia democrática. Finalmente, se incluye una nota acerca de la oferta de servicios colectivos por parte del Estado en España para completar la información sobre el tema.

Todos estos trabajos muestran que no se han modificado los componentes patriarcales de la familia y la sociedad, que no se han podido revalorizar los servicios prestados por la mujer en la familia y que se ha producido una deshumanización de la sociedad en conjunto. En este contexto, la familia igualitaria ha entrado también en crisis. Esta crisis y su vinculación con la crisis del Estado es uno de los problemas contemporáneos cuyo análisis parece importante desde una perspectiva teórica y desde una perspectiva política.

Judith Astelarra y M. Jesús Izquierdo 